\title{
Multiplier ideal sheaves and the Kähler-Ricci flow on toric Fano manifolds with large symmetry
}

\author{
YUJI SANO
}

The purpose of this paper is to calculate the support of the multiplier ideal subschemes derived from the Kähler-Ricci flow on certain toric Fano manifolds with large symmetry. The early idea of this paper has already been in the Appendix of [12].

\section{Introduction}

In [12], Futaki and Sano investigate the relationship between the multiplier ideal subvariety derived from the continuity method on toric Fano manifolds and Futaki invariant, and calculate the multiplier ideal subvariety on a simple example. On the other hand, the relationship between the multiplier ideal sheaves and the Kähler-Ricci flow has recently been studied. The first work on this topic is given by Phong-Sesum-Sturm [20]. They give a sufficient and necessary condition for the convergence of the Kähler-Ricci flow in the terms of the multiplier ideal sheaves. After [20], Rubinstein [21] proves that the Kähler-Ricci flow will induce a multiplier ideal sheaf satisfying the same properties as Nadel's multiplier ideal sheaves derived from the continuity method. The purpose of this paper is to calculate the multiplier ideal subvarieties from the Kähler-Ricci flow in the sense of [21] on certain toric Fano manifolds with large symmetry. Our method owes largely to the result about the convergence of the Kähler-Ricci flow on toric Fano manifolds due to Zhu [31].

Let $(X, \omega)$ be an $n$-dimensional Fano manifold with a Kähler form $\omega$ representing $c_{1}(X)$. The normalized Kähler-Ricci flow on $X$ is defined by

$$
\frac{d}{d t} \omega_{t}=-\operatorname{Ric}\left(\omega_{t}\right)+\omega_{t}
$$

where $t \in \mathbb{R}_{\geq 0}, \operatorname{Ric}\left(\omega_{t}\right)$ is the Ricci form of $\omega_{t}$ and $\omega_{0}=\omega$. Since the flow (1.1) preserves the Kähler class, we can consider the corresponding equation 
to (1.1) with respect to Kähler potentials

$$
\left\{\begin{array}{l}
\frac{\partial \varphi_{t}}{\partial t}=\log \frac{\operatorname{det}\left(g_{i \bar{j}}+\varphi_{i \bar{j}}\right)}{\operatorname{det}\left(g_{i \bar{j}}\right)}+\varphi_{t}-h_{0}, \\
\varphi_{0} \equiv c_{0}
\end{array}\right.
$$

where $\omega_{t}=\omega_{0}+\frac{\sqrt{-1}}{2 \pi} \partial \bar{\partial} \varphi_{t}, c_{0}$ is a constant and $h_{0}$ is a real-valued function determined by

$$
\operatorname{Ric}\left(\omega_{0}\right)-\omega_{0}=\frac{\sqrt{-1}}{2 \pi} \partial \bar{\partial} h_{0}, \int_{X} \mathrm{e}^{h_{0}} \omega_{0}^{n}=V .
$$

Here we denote the volume of $X$ with respect to $\omega_{0}$ by $V$. The existence of the solution of (1.2) for all $t>0$ is proved by Cao [3] by following Yau's argument in [30]. Since there are some obstructions for the existence of KählerEinstein metrics on Fano manifolds [11, 15, 26], the Kähler-Ricci flow does not necessarily converge on Fano manifolds. Nadel [16] and Demailly and Kollár [7] prove that if $X$ does not admit Kähler-Einstein metrics, then the failure of the closedness condition for the continuity method induces a multiplier ideal sheaf. (This fact can be extended in the cases of other canonical Kähler metrics such as Kähler-Ricci solitons [12] and Kähler-Einstein metrics in the sense of Mabuchi [22].) The analogous result for the Kähler-Ricci flow is proved recently by Rubinstein [21]. In this paper, we consider the multiplier ideal sheaves in the sense of [7]. Let $\psi$ be an almost plurisubharmonic function (psh function) on $X$, i.e., $\psi$ is written locally as a sum of a psh function and a smooth function. For $\psi$, we define the multiplier ideal sheaf $\mathcal{I}(\psi) \subset \mathcal{O}_{X}$ as follows; for every open subset $U \subset X$, the space $\Gamma(U, \mathcal{I}(\psi))$ of local sections of $\mathcal{I}(\psi)$ over $U$ is given by

$$
\Gamma(U, \mathcal{I}(\psi))=\left\{\left.f \in \mathcal{O}_{X}(U)\left|\int_{U}\right| f\right|^{2} \mathrm{e}^{-\psi} d \nu<\infty\right\},
$$

where $f$ is a holomorphic function on $U$ and $d \nu$ is a fixed volume form on $X$. Note that $\mathcal{I}(\psi)$ is a coherent ideal sheaf (cf.[7]) and invariant up to an additive constant. The result of [21] is as follows.

Theorem 1.1 [21]. Let $(X, \omega)$ be an n-dimensional Fano manifold with a Kähler form $\omega$ in $c_{1}(X)$, and $G \subset A$ A $(X)$ be a compact subgroup of the group Aut $(X)$ of holomorphic automorphisms of $X$. Let $\gamma \in(n /(n+1), 1)$. Suppose that $X$ does not admit Kähler-Einstein metrics. Then there is an initial condition $\varphi_{0} \equiv c_{0}$ in (1.2) and a sequence $\left\{\varphi_{t_{j}}\right\}_{j \geq 0}$ such that $\varphi_{t_{j}}-\sup \varphi_{t_{j}}$ 
converges to an almost psh function $\varphi_{\infty}$ in $L^{1}$-topology and the associated multiplier ideal sheaf $\mathcal{I}\left(\gamma \varphi_{\infty}\right)$ is $G^{\mathbb{C}}$-invariant and proper, i.e., $\mathcal{I}\left(\gamma \varphi_{\infty}\right)$ is equal neither to 0 nor $\mathcal{O}_{X}$, where $G^{\mathbb{C}}$ is the complexification of $G$.

Remark that in [21] the multiplier ideal sheaf is constructed from the sequence of $\left\{\varphi_{t}-\frac{1}{V} \int_{X} \varphi_{t} \omega^{n}\right\}_{t}$ instead of $\left\{\varphi_{t}-\sup \varphi_{t}\right\}_{t}$ but there is no difference between them due to a standard argument by the Green function, more precisely, there is a constant $C$ such that $\sup \varphi_{t}-C \leq \int_{X} \varphi_{t} \omega^{n} \leq$ $\sup \varphi_{t}$. For $\gamma \in(0,1)$, we denote the subscheme of $X$ cut out by $\mathcal{I}\left(\gamma \varphi_{\infty}\right)$ by $V_{\gamma}$. In this paper, we call it the KRF-multiplier ideal subscheme (KRFMIS) of exponent $\gamma$. We often abbreviate the subschemes cut out by (general) multiplier ideal sheaves to the MIS. In particular, for an almost psh function $\varphi$ we call the subscheme cut out by $\mathcal{I}(\gamma \varphi)$ the MIS of exponent $\gamma$ (with respect to $\varphi$ ). The exponent of the MIS is closely related to the complex singularity exponent, which is introduced by Demailly-Kollár [7]. The definition of the complex singularity exponent will be explained in Section 3. Here let us remark that the complex singularity exponent is a local version of a holomorphic invariant, which is called the $\alpha$-invariant defined by Tian [24]. In fact, Theorem 1.1 in [21] is obtained by effectively proving that if $\alpha_{G}(X)>\frac{n}{n+1}$ then the Kähler-Ricci flow converges. Remark that $\alpha_{G}(X) \geq 1$ if there is no multiplier ideal sheaf $\mathcal{I}(\psi)$ such that there is a positive constant $\varepsilon$ satisfying that $\mathcal{I}(\gamma \psi)$ is proper for $\gamma \in(1-\varepsilon, 1)$. See [2, $4,5,9,13,23,25]$ for other works related to the $\alpha$-invariant.

On the other hand, it has been conjectured that the existence of KählerEinstein metrics would be equivalent to certain stability of manifolds in the sense of Geometric Invariant Theory (cf.[8],[26]). In this viewpoint, we expect that the KRF-MIS would be related to stability of manifolds. Hence, concrete calculations of the KRF-MIS would be useful to verify the expectation. Our main result is as follows.

Theorem 1.2. Let $X$ be a toric Fano manifold in $\mathcal{W}_{1}$. Suppose that $X$ does not admit Kähler-Einstein metrics. Let $v_{\mathrm{KRS}}$ be the holomorphic vector field for a Kähler-Ricci soliton on $X$. Suppose that the imaginary part of $v_{\mathrm{KRS}}$ generates a one-parameter subgroup of a compact subgroup $G$ (defined in Section 2) of $\operatorname{Aut}(X)$. Let $\left\{\sigma_{t}:=\exp \left(t v_{\mathrm{KRS}}\right)\right\}$ and $\gamma \in(0,1)$. Then, there exists a subsequence of $\left\{t_{j}\right\}$ in Theorem 1.1 such that the support of the induced KRF-MIS of exponent $\gamma$ is equal to the support of the MIS of exponent $\gamma$ derived from a sequence of Kähler potentials of $\left\{\left(\sigma_{t_{j}}^{-1}\right)^{*} \omega\right\}$ for any G-invariant Kähler form $\omega$. 
The definitions of $\mathcal{W}_{1}$ and Kähler-Ricci solitons will be explained in Section 2. In [30], it is proved that a Kähler-Ricci soliton always exists on any toric Fano manifold.

Remark 1.1. The author expects that the restriction to $\mathcal{W}_{1}$ would be just a technical assumption and it would be removed. ${ }^{1}$

We can calculate the support of the KRF-MIS concretely by using Theorem 1.2 and a formula in Theorem 3.1 to compute the complex singularity exponent of the almost psh function induced from one-parameter subgroups of the torus action. For example,

Corollary 1.1. Let $X$ be the blow up of $\mathbb{C P}^{2}$ at $p_{1}$ and $p_{2}$. Let $E_{1}$ and $E_{2}$ be the exceptional divisors of the blow up, and $E_{0}$ be the proper transform of $\overline{p_{1} p_{2}}$ of the line passing through $p_{1}$ and $p_{2}$. Then, the support of the KRFMIS on $X$ of exponent $\gamma$ is

$$
\left\{\begin{array}{cc}
\cup_{i=0}^{2} E_{i} & \text { for } \gamma \in\left(\frac{1}{2}, 1\right), \\
E_{0} & \text { for } \gamma \in\left(\frac{1}{3}, \frac{1}{2}\right) .
\end{array}\right.
$$

The organization of this paper is as follows. In Section 2, we reduce the KRF-MIS to a simpler one by following the proof of [31]. In Section 3, we give the formula to calculate the complex singularity exponent of the associated almost psh function derived from one-parameter subgroups of the torus action and complete the proof of Theorem 1.2. In Section 4, we calculate examples of toric Fano $n$-folds $(n=2,3)$ contained in $\mathcal{W}_{1}$ by using our results.

\section{Convergence of the Kähler-Ricci flow to Kähler-Ricci solitons on toric Fano manifolds}

Through this section and the next section, we prove Theorem 1.2 by following the proof of [31].

First, we explain the setup concerning toric Fano manifolds briefly. (Consult [18] and [10] for general information of toric geometry.) A toric variety

\footnotetext{
${ }^{1}$ The restriction to $\mathcal{W}_{1}$ does not imply the non-existence of Kähler-Einstein metrics. In fact, examples of Kähler-Einstein toric Fano manifolds in $\mathcal{W}_{1}$ are found (cf. $[17,19])$.
} 
$X$ is an algebraic variety with an effective action of $T_{\mathbb{C}}:=\left(\mathbb{C}^{*}\right)^{n}$, where $\operatorname{dim}_{\mathbb{C}} X=n$. Let $T_{\mathbb{R}}:=\left(S^{1}\right)^{n}$ be the real torus in $T_{\mathbb{C}}$ and $\mathfrak{t}_{\mathbb{R}}$ be the associated Lie algebra. Let $N_{\mathbb{R}}:=J \mathfrak{t}_{\mathbb{R}} \simeq \mathbb{R}^{n}$ where $J$ is the complex structure of $T_{\mathbb{C}}$. Let $M_{\mathbb{R}}$ be the dual space $\operatorname{Hom}\left(N_{\mathbb{R}}, \mathbb{R}\right) \simeq \mathbb{R}^{n}$ of $N_{\mathbb{R}}$. Denoting the group of algebraic characters of $T_{\mathbb{C}}$ by $M$, then $M_{\mathbb{R}}=M \otimes_{\mathbb{Z}} \mathbb{R}$. Let $P^{*}$ be the image of $X$ under the moment map, which is a convex reflexive polytope. Let $\mathrm{L} P^{*}=\left\{p^{(i)}\right\}_{1 \leq i \leq m}$ be the set of all lattice points contained in $P^{*}$. Let $P$ be the dual polytope defined by

$$
P=\left\{x \in N_{\mathbb{R}} \mid\langle p, x\rangle \leq 1 \text { for any vertex } p \text { of } P^{*}\right\}
$$

where $\langle\cdot, \cdot\rangle$ is the natural pairing on $M_{\mathbb{R}} \times N_{\mathbb{R}}$. The polytope $P$ is often called the Fano polytope of $X$.

Let $\mathcal{N}\left(T_{\mathbb{C}}\right)$ be the normalizer of $T_{\mathbb{C}}$ in $\operatorname{Aut}(X)$. Then the Weyl group $\mathcal{W}(X):=\mathcal{N}\left(T_{\mathbb{C}}\right) / T_{\mathbb{C}}$ of $\operatorname{Aut}(X)$ with respect to $T_{\mathbb{C}}$ is equal to the finite subgroup of $\mathrm{GL}(n, \mathbb{Z})$ consisting of all elements, which preserve $P$ where $N \simeq \mathbb{Z}^{n}$ is the dual of $M$ (Proposition 3.1 in [2]). We define $G$ in Theorem 1.2 by the compact subgroup of $\operatorname{Aut}(X)$ generated by $T_{\mathbb{R}}$ and $\mathcal{W}(X)$. Let $N_{\mathbb{R}}^{\mathcal{W}(X)}:=\left\{x \in N_{\mathbb{R}} \mid x^{g}=x\right.$ for all $\left.g \in \mathcal{W}(X)\right\}$. Then, the class of toric Fano manifolds, which we shall consider is

$$
\mathcal{W}_{1}:=\left\{X: \text { toric Fano manifold with } \operatorname{dim} N_{\mathbb{R}}^{\mathcal{W}(X)}=1\right\}
$$

As an initial Kähler form $\omega_{0}$ on $X$, we take a standard metric determined by the moment polytope $P^{*}$ as follows. Let $\left(\frac{1}{2} x_{1}+\sqrt{-1} \theta_{1}, \ldots, \frac{1}{2} x_{n}+\right.$ $\left.\sqrt{-1} \theta_{n}\right)$ be an affine logarithm coordinates on $T_{\mathbb{C}}=T_{\mathbb{R}} \times N_{\mathbb{R}}$, i.e., $t_{i}=$ $\exp \left(\frac{1}{2} x_{i}+\sqrt{-1} \theta_{i}\right)$ where $t=\left(t_{1}, \ldots, t_{n}\right) \in T_{\mathbb{C}}$. By the invariance, we define $\omega_{0}:=\frac{\sqrt{-1}}{2 \pi} \partial \bar{\partial} u_{0}$ on a dense orbit of the action of $T_{\mathbb{C}}$, where $u_{0}$ is also a convex function on $N_{\mathbb{R}}$ defined by

$$
u_{0}(x):=\log \left(\sum_{i=1}^{m} \mathrm{e}^{\left\langle p^{(i)}, x\right\rangle}\right)
$$

and $x=\left(x_{1}, \ldots, x_{n}\right) \in N_{\mathbb{R}}$. It is known that $\omega_{0}$ can be extended to a welldefined Kähler form on $X$. Obviously $\omega_{0}$ and $u_{0}$ are $\mathcal{W}(X)$-invariant.

Now, we begin the proof of Theorem 1.2. The proof is based on the following result concerning the convergence of the Kähler-Ricci flow. Recall that a pair $\left(v_{\mathrm{KRS}}, \omega_{\mathrm{KRS}}\right)$ of a holomorphic vector field and a Kähler form on a Fano manifold is called a Kähler-Ricci soliton if

$$
\operatorname{Ric}\left(\omega_{\mathrm{KRS}}\right)-\omega_{\mathrm{KRS}}=\mathcal{L}_{v_{\mathrm{KRS}}} \omega_{\mathrm{KRS}}
$$


where $\mathcal{L}_{v}$ is the Lie derivative along $v$. Let $\operatorname{Aut}_{r}(X)$ be the reductive part of $\operatorname{Aut}(X)$ and $K$ be a maximal compact subgroup of $\operatorname{Aut}_{r}(X)$. Note that $\operatorname{Aut}_{r}(X)$ is the complexification of $K$. From the uniqueness of Kähler-Ricci solitons proved by Tian-Zhu in [27], we may assume that a Kähler-Ricci soliton $\left(v_{\mathrm{KRS}}, \omega_{\mathrm{KRS}}\right)$ is $K$-invariant and the imaginary part of $v_{\mathrm{KRS}}$ generates a one-parameter subgroup $K_{v_{\text {KRS }}}$ of $K$. For a holomorphic vector field $v$, let $F_{v}$ be the holomorphic invariant of Tian-Zhu [27], which is a generalization of Futaki invariant. Then $v_{\mathrm{KRS}}$ satisfies that $F_{v_{\mathrm{KRS}}}$ vanishes on $\operatorname{Aut}_{r}(X)$.

Theorem 2.1 Zhu, [31]. On a toric Fano manifold $X$, the normalized Kähler-Ricci flow with any $T_{\mathbb{R}}$-invariant Kähler metric converges to a Kähler-Ricci soliton $\omega_{\mathrm{KRS}}$ in the sense of Cheeger-Gromov.

This is a toric version of [28]. Theorem 2.1 indicates that the asymptotic behavior of $\omega_{t}$ in (1.1) is similar to $\left(\sigma_{t}^{-1}\right)^{*} \omega_{\mathrm{KRS}}$ where $\sigma_{t}=\exp \left(t v_{\mathrm{KRS}}\right)$. Indeed, $\left\{\left(\sigma_{t}^{-1}\right)^{*} \omega_{\mathrm{KRS}}\right\}_{t}$ satisfies equation (1.1). The key of the proof of Theorem 1.2 is to estimate the difference between $\omega_{t}$ in $(1.1)$ and $\left(\sigma_{t}^{-1}\right)^{*} \omega_{\mathrm{KRS}}$. So, we recall a part of [31] needed for our proof.

Let us consider the equation of (1.2) with the initial metric $\omega_{0}$ defined by using $u_{0}$ in (2.1) and $c_{0}=0$. Remark that the assumption of $c_{0}$ is different from the initial constant in [21], but we shall see in the proof of Lemma 2.1 that this difference does not affect the KRF-MIS. Since all objects concerning (1.2) are $T_{\mathbb{R}}$-invariant, we can reduce (1.2) to a real Monge-Ampère equation

$$
\left\{\begin{array}{l}
\frac{\partial u}{\partial t}=\log \operatorname{det}\left(u_{i j}\right)+u \\
u(0, \cdot)=u_{0}
\end{array}\right.
$$

where $u_{t}=u(t, \cdot)=u_{0}+\varphi_{t}$ on $N_{\mathbb{R}}$. Here we reduce the potential function $\varphi_{t}$ of $\omega_{t}$ to a function on $N_{\mathbb{R}}$ by the invariance under $T_{\mathbb{R}}$-action. To avoid the complication of symbols, we denote it by the same $\varphi_{t}$. Note that $\varphi_{t}$ on $N_{\mathbb{R}}$ is normalized by requiring that the image of the gradient map of $u_{t}$ in $M_{\mathbb{R}}$ is equal to $P^{*}$. For each solution $u_{t}$ of $(2.2)$, let $x_{t}$ be the minimal point of $u_{t}$. The assumption that $X$ is contained in $\mathcal{W}_{1}$ makes the behavior of $\left\{x_{t}\right\}_{t}$ simple. Let $\beta_{\mathrm{KRS}}$ be the vector in $N_{\mathbb{R}}$, which induces the holomorphic vector field $v_{\mathrm{KRS}}$ of the Kähler-Ricci soliton. More precisely, if $v_{\mathrm{KRS}}^{\sharp}$ is the real vector field induced by $\beta_{\mathrm{KRS}}$ then $v_{\mathrm{KRS}}=\frac{1}{2}\left(v_{\mathrm{KRS}}^{\sharp}-\sqrt{-1}\left(J v_{\mathrm{KRS}}^{\sharp}\right)\right)$. Since $\beta_{\mathrm{KRS}}$ is $\mathcal{W}(X)$-invariant and $X \in \mathcal{W}_{1}$, the line $\left\{s \beta_{\text {KRS }} \mid s \in \mathbb{R}\right\}$ is equal to the fixed subspace of $N_{\mathbb{R}}$ under the action of $\mathcal{W}(X)$. Since $u_{t}$ is also $\mathcal{W}(X)$-invariant, $\left\{x_{t}\right\}_{t}$ is contained in the line $\left\{s \beta_{\mathrm{KRS}} \mid s \in \mathbb{R}\right\}$, that is to say, for each $t$ there 
is a constant $s_{t} \in \mathbb{R}$ such that $x_{t}=s_{t} \beta_{\mathrm{KRS}}$. Remark that $\left|d s_{t} / d t\right|$ is uniformly bounded for all $t$, which is due to Lemma 4.6 [6] and Lemma 4.1 [31].

Let $\rho_{t}$ be a holomorphic transformation, which induces the shift transformation on $N_{\mathbb{R}}$ defined by $x \mapsto x+s_{t} \beta_{\mathrm{KRS}}$ for each $t$. Let $\tilde{\varphi}_{t}$ be a Kähler potential defined by

$$
\rho_{t}^{*} \omega_{\varphi_{t}}=\omega_{0}+\frac{\sqrt{-1}}{2 \pi} \partial \bar{\partial} \tilde{\varphi}_{t}
$$

which is what we desire. Remark that $\tilde{\varphi}_{t}$ is equal to $u_{t}\left(\cdot+s_{t} \beta_{\mathrm{KRS}}\right)-u_{0}(\cdot)$ up to constant. The ambiguity of an additive constant in (2.3) is removed by requiring

$$
\frac{\partial \tilde{\varphi}_{t}}{\partial t}=\log \frac{\operatorname{det}\left(g_{i \bar{j}}+\tilde{\varphi}_{i \bar{j}}\right)}{\operatorname{det}\left(g_{i \bar{j}}\right)}+\tilde{v}_{t}\left(\tilde{\varphi}_{t}\right)+\tilde{\varphi}_{t}-\tilde{h}_{0}+\theta_{\tilde{v}_{t}}
$$

on $X$, where

$$
\tilde{v}_{t}:=\frac{d x_{t}}{d t}=\beta_{\mathrm{KRS}} \cdot \frac{d s_{t}}{d t}
$$

$\theta_{\tilde{v}_{t}}:=\tilde{v}_{t}\left(u_{0}\right)$. The function $\tilde{h}_{0}$ is the renormalized function of $h_{0}$ as follows. Let $V$ be the volume of $X$ with respect to $\omega_{0}$. Let $\theta_{v_{\mathrm{KRS}}}=v_{\mathrm{KRS}}\left(u_{0}\right)$ and $\theta_{\tilde{v}_{t}}:=\tilde{v}_{t}\left(u_{0}\right)$. Let $\varphi_{t}^{\prime}$ be the Kähler potential defined by

$$
\sigma_{t}^{*} \omega_{\varphi_{t}}=\omega_{0}+\frac{\sqrt{-1}}{2 \pi} \partial \bar{\partial} \varphi_{t}^{\prime}
$$

and

$$
\frac{\partial \varphi_{t}^{\prime}}{\partial t}=\log \frac{\operatorname{det}\left(g_{i \bar{j}}+\left(\varphi_{t}^{\prime}\right)_{i \bar{j}}\right)}{\operatorname{det}\left(g_{i \bar{j}}\right)}+v_{\mathrm{KRS}}\left(\varphi_{t}^{\prime}\right)+\varphi_{t}^{\prime}-h_{0}+\theta_{v_{\mathrm{KRS}}} .
$$

Then $\tilde{h}_{0}$ satisfies

$$
\begin{aligned}
\frac{1}{V} \int_{X}\left(\tilde{h}_{0}-\theta_{v_{\mathrm{KRS}}}\right) \omega_{0}^{n}= & -\frac{1}{V} \int_{0}^{\infty} \int_{X}\left\|\bar{\partial} \frac{\partial \varphi_{t}^{\prime}}{\partial t}\right\|^{2} \exp \left(\theta_{v_{\mathrm{KRS}}}+v_{\mathrm{KRS}}\left(\varphi_{t}^{\prime}\right)-t\right) \\
& \wedge\left(\sigma_{t}^{*} \omega_{\varphi_{t}}\right)^{n} \wedge d t
\end{aligned}
$$

as Proposition 3.2 [31]. Then, it is proved

Proposition 2.1 [31]. The family $\left\{\omega_{\tilde{\varphi}_{t}}\right\}_{t}$ converges to a Kähler-Ricci soliton associated to $v_{\mathrm{KRS}}$ and $\tilde{v}_{t}$ converges to $v_{\mathrm{KRS}}$ as $t$ goes to infinity. 
Remark that $\frac{d s_{t}}{d t} \rightarrow 1$ as $t \rightarrow \infty$, because $\tilde{v}_{t}$ converges to $v_{\mathrm{KRS}}$. Therefore we can conclude the following lemma. Let $\psi_{t}$ be the function defined by

$$
\left(\rho_{t}^{-1}\right)^{*} \omega=\omega+\frac{\sqrt{-1}}{2 \pi} \partial \bar{\partial} \psi_{t}, \sup \psi_{t}=0,
$$

where $\omega$ is a $G$-invariant Kähler form. By replacing the sequence $\left\{t_{j}\right\}$ in Theorem 1.1 by an appropriate subsequence if necessary, we have the limit of $\left\{\psi_{t_{j}}\right\}$ in $L^{1}$-topology. We denote it by $\psi_{\infty}$. Remark that we replace $\varphi_{\infty}$ in Theorem 1.1 by the one with respect to the above new sequence $\left\{t_{j}\right\}$.

Lemma 2.1. $\mathcal{I}\left(\gamma \varphi_{\infty}\right)$ and $\mathcal{I}\left(\gamma \psi_{\infty}\right)$ coincide for any $\gamma \in(0,1)$.

Proof. First, we shall see that the difference of the choice of initial constant $c_{0}$ does not matter when we consider the KRF-MIS in the sense of [21]. Let $\varphi_{1, t}$ and $\varphi_{2, t}$ be the solutions of (1.2) with different initial constants $c_{1}$ and $c_{2}$. Then, $\varphi_{2, t}=\varphi_{1, t}-\left(c_{1}-c_{2}\right) \mathrm{e}^{t}$. Since $\varphi_{1, t}-\sup \varphi_{1, t}$ is equal to $\varphi_{2, t}-\sup \varphi_{2, t}$ for each $t$, their MIS coincide.

The KRF-MIS is independent of the choice of $\omega$ in (2.5). Take any $G$ invariant Kähler form $\omega$. As seen in the above argument, we find that $\omega_{\varphi_{t}}$ is equal to $\left(\rho_{t}^{-1}\right)^{*} \omega_{\tilde{\varphi}_{t}}$ for each $t$. Let $\phi_{t} \in C^{\infty}(X)$ be the discrepancy function defined by $\omega_{\tilde{\varphi}_{t}}-\omega=\frac{\sqrt{-1}}{2 \pi} \partial \bar{\partial} \phi_{t}$ and $\sup \phi_{t}=0$. Since $\omega_{\tilde{\varphi}_{t}}$ converges in $C^{\infty}$ sense, $\left\|\phi_{t}\right\|_{C^{0}}$ is uniformly bounded. Then

$$
\psi_{t}=\left(\varphi_{t}-\left(\rho_{t}^{-1}\right)^{*} \phi_{t}\right)-\sup \left(\varphi_{t}-\left(\rho_{t}^{-1}\right)^{*} \phi_{t}\right) .
$$

Since $\left\|\left(\rho_{t}^{-1}\right)^{*} \phi_{t}\right\|_{C^{0}}$ is also uniformly bounded, the MIS of $\left\{\psi_{t_{j}}\right\}$ and of $\left\{\varphi_{t_{j}}-\right.$ $\left.\sup \varphi_{t_{j}}\right\}$ coincide. The proof is completed.

\section{Complex singularity exponents of multiplier ideal sheaves on toric Fano manifolds}

In this section, we prove the following lemma to finish the proof of Theorem 1.2. Let $\overline{\varphi_{t}}$ be the Kähler potential defined by

$$
\left(\sigma_{t}^{-1}\right)^{*} \omega=\omega+\frac{\sqrt{-1}}{2 \pi} \partial \bar{\partial} \bar{\varphi}_{t}, \sup \bar{\varphi}_{t}=0,
$$

where $\omega$ is a $G$-invariant Kähler form. By replacing $\left\{t_{j}\right\}$ in Theorem 1.1 again by its appropriate subsequence if necessary, we have the limit of $\left\{\bar{\varphi}_{t_{j}}\right\}$ in $L^{1}$-topology. We denote it by $\bar{\varphi}_{\infty}$. Remark that we replace $\varphi_{\infty}$ again by the one with respect to the new subsequence of $\left\{t_{j}\right\}$. 
Lemma 3.1. For any $\gamma \in(0,1)$, the support of the MIS of exponent $\gamma$ with respect to $\psi_{\infty}$ is equal to the one with respect to $\bar{\varphi}_{\infty}$.

To prove Lemma 3.1, we shall give a formula to calculate the complex singularity exponent of $\psi_{\infty}$ with respect to each face of the polytope $P^{*}$. Furthermore, we give a way to determine the support of the KRF-MIS. We should note that the assumption on $\mathcal{W}_{1}$ is not needed for the argument in this section.

First, let us recall the complex singularity exponent. Let $X$ be a complex manifold and $\varphi$ be an almost psh function on $X$. Let $K \subset X$ be a compact subset of $X$. The complex singularity exponent $c_{K}(\varphi)$ of $\varphi$ on $K$ is defined by

$$
c_{K}(\varphi):=\sup \left\{c \geq 0 ; \exp (-c \varphi) \text { is } L^{1} \text { on a neighborhood of } K\right\} .
$$

From its definition, $c_{\{p\}}(\varphi)$ is strictly less than some positive constant $\gamma$ if and only if the local section $1_{U_{p}}$ of $\mathcal{O}_{X}\left(U_{p}\right)$ is not contained in $\Gamma\left(U_{p}, \mathcal{I}(\gamma \varphi)\right)$ for any open neighborhood $U_{p}$ at $p$, i.e., $p$ is contained in the support of the subscheme cut out by $\mathcal{I}(\gamma \varphi)$. That is to say, the support of the MIS of exponent $\gamma$ with respect to $\varphi$ is equal to $\left\{p \in X \mid c_{\{p\}}(\varphi)<\gamma\right\}$.

From now on, let $X$ be a toric Fano manifold. Let $\psi_{t}$ and $\psi_{\infty}$ be as before. We take the standard Kähler form $\omega_{0}$ using by (2.1) as $\omega$ in (2.5). For a point $y \in P^{*}$, we denote the complex singularity exponent of $\psi_{\infty}$ on $\mu^{-1}(y)$ by $c_{\{y\}}\left(\psi_{\infty}\right)$ where $\mu$ is the moment map with respect to $\omega_{0}$. This notation makes sense. In fact, $\mu^{-1}(y)$ is contained in the support of the MIS of exponent $\gamma$ with respect to $\psi_{\infty}$ if and only if $c_{\{y\}}\left(\psi_{\infty}\right)<\gamma$, because the MIS on a toric manifold is $T_{\mathbb{R}}$-invariant and

$$
c_{\{y\}}\left(\psi_{\infty}\right)=\inf _{p \in \mu^{-1}(y)} c_{\{p\}}\left(\psi_{\infty}\right)
$$

It is easy to check as follows. It is trivial that $c_{\{y\}}\left(\psi_{\infty}\right) \leq \inf _{p \in \mu^{-1}(y)} c_{\{p\}}\left(\psi_{\infty}\right)$ from the definition. For any $c<\inf _{p \in \mu^{-1}(y)} c_{\{p\}}\left(\psi_{\infty}\right)$, there is an open covering $\cup_{p \in \mu^{-1}(y)} U_{p}$ of $\mu^{-1}(y)$ such that $U_{p}$ is an open neighborhood at $p$ and $\mathrm{e}^{-c \psi_{\infty}}$ is integrable over $U_{p}$. Since $\mu^{-1}(y)$ is compact, we find that $\mathrm{e}^{-c \psi_{\infty}}$ is integrable over $\cup_{p \in \mu^{-1}(y)} U_{p}$, i.e., $c \leq c_{\{y\}}\left(\psi_{\infty}\right)$. Hence (3.1) is proved.

For each face $\delta^{*}$ of $P^{*}$, let us calculate $c_{\{y\}}\left(\psi_{\infty}\right)$ where $y$ is a point in the relative interior of $\delta^{*}$. In order to do it, we shall choose a reference point in the interior of $\delta^{*}$ as follows. Let $\delta^{*}$ be an $(n-l-1)$-dimensional face of $P^{*}$. Let $\tilde{\mu}$ be the $G$-equivariant moment map from $N_{\mathbb{R}}$ to $M_{\mathbb{R}}$ with respect 
to $\omega_{0}$ defined by

$$
\tilde{\mu}(x):=\left(\frac{\partial u_{0}}{\partial x_{1}}(x), \ldots, \frac{\partial u_{0}}{\partial x_{n}}(x)\right)
$$

where $u_{0}$ is defined by (2.1). Remark that the image of $\tilde{\mu}$ is equal to the interior of $P^{*}$. From the duality between $P$ and $P^{*}$, for $\delta^{*}$ there is a unique $l$-dimensional face $\delta$ of $P$. Let $\left\{q^{(i)}\right\}_{i=1, \ldots, l+1}$ be the set of vertices of $\delta$. For $a_{i} \in \mathbb{R}_{>0}$ satisfying $\sum_{i=1}^{l+1} a_{i}=1$, we put $x^{(a)}:=a_{1} q^{(1)}+\cdots+a_{l+1} q^{(l+1)}$. Obviously $x^{(a)}$ is contained in the relative interior of $\delta$. Then

$$
\begin{aligned}
\frac{\partial u_{0}}{\partial x_{j}}\left(s x^{(a)}\right) & =\frac{1}{\sum_{i=1}^{m} \mathrm{e}^{\left\langle p^{(i)}, s x^{(a)}\right\rangle}}\left\{\sum_{i=1}^{m} p_{j}^{(i)} \mathrm{e}^{\left\langle p^{(i)}, s x^{(a)}\right\rangle}\right\} \\
& =\frac{1}{\left(\sum_{i_{\alpha} \in A} \mathrm{e}^{s}\right)+o\left(\mathrm{e}^{s}\right)}\left\{\mathrm{e}^{s}\left(\sum_{i_{\alpha} \in A} p_{j}^{\left(i_{\alpha}\right)}\right)+o\left(\mathrm{e}^{s}\right)\right\} \\
& \rightarrow \frac{\sum_{i_{\alpha} \in A} p_{j}^{\left(i_{\alpha}\right)}}{\sharp A}
\end{aligned}
$$

as $s \rightarrow \infty$, where $A$ is a subset of $\{1, \ldots, m\}$ such that $i_{\alpha} \in A$ if and only if $p^{\left(i_{\alpha}\right)}$ is contained in $\cap_{i=1}^{l+1} H_{i}$, where $H_{i}:=\left\{y \in M_{\mathbb{R}} \mid\left\langle y, q^{(i)}\right\rangle=1\right\}$. In the above $f(s) \in o\left(\mathrm{e}^{c s}\right)$ means $\lim _{s \rightarrow \infty} f(s) \mathrm{e}^{-c s}=0$ and $\sharp A$ denotes the number of integers in $A$. Equation (3.2) means that the point

$$
p^{\left(\delta^{*}\right)}:=\lim _{s \rightarrow \infty} \tilde{\mu}\left(s x^{(a)}\right)
$$

is independent of the choice of a vector $a$ and is contained in the relative interior of the face $\delta^{*}$. In fact, $\left\{p^{\left(i_{\alpha}\right)}\right\}_{i_{\alpha} \in A}$ is the set of all integral points on $\delta^{*}$ and $p^{\left(\delta^{*}\right)}$ is the average of them. So, in order to determine whether $\mu^{-1}\left(\delta^{*}\right)$ is contained in the MIS of exponent $\gamma$ or not, it is sufficient to determine whether $c_{\left\{p^{\left(\delta^{*}\right)}\right\}}\left(\psi_{\infty}\right)$ is strictly smaller than $\gamma$ or not. In fact, the

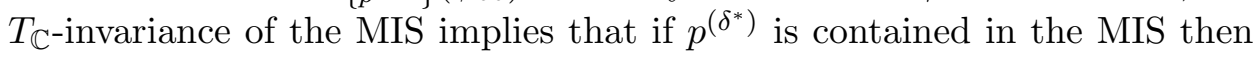
$\delta^{*}$ is also contained in it.

Next, we shall give a formula to calculate $c_{\left\{p^{\left(\delta^{*}\right)}\right\}}\left(\psi_{\infty}\right)$ for each face $\delta^{*}$ of $P^{*}$. Let $\mathrm{L} P_{\max }^{*}$ be the set of all integral points $p$ contained in $P^{*}$ satisfying

$$
\left\langle p,-\beta_{\mathrm{KRS}}\right\rangle=\max _{p^{(i)} \in \mathrm{L} P^{*}}\left\langle p^{(i)},-\beta_{\mathrm{KRS}}\right\rangle .
$$


Let $u_{0}^{\prime}(t, x)$ be a convex function on $N_{\mathbb{R}}$ defined by

$$
u_{0}^{\prime}(t, x):=\log \left(\sum_{i=1}^{m} \mathrm{e}^{\left\langle p^{(i)}, x-s_{t} \beta_{\mathrm{KRS}}\right\rangle}\right)-s_{t} \max _{i=1, \ldots, m}\left\langle p^{(i)},-\beta_{\mathrm{KRS}}\right\rangle
$$

Then, $\left(\rho_{t}^{-1}\right)^{*} \omega_{0}=\frac{\sqrt{-1}}{2 \pi} \partial \bar{\partial} u_{0}^{\prime}(t, x)$. We find

$$
\begin{aligned}
u_{0}^{\prime}(t, x)-u_{0}(x) & =\log \left(\frac{\sum_{i=1}^{m} \mathrm{e}^{\left\langle p^{(i)}, x\right\rangle+s_{t}\left(\left\langle p^{(i)},-\beta_{\mathrm{KRS}}\right\rangle-\max _{j}\left\langle p^{(j)},-\beta_{\mathrm{KRS}}\right\rangle\right)}}{\sum_{i=1}^{m} \mathrm{e}^{\left\langle p^{(i)}, x\right\rangle}}\right) \\
& \leq 0
\end{aligned}
$$

for all $x \in N_{\mathbb{R}}$ and all $t \in \mathbb{R}_{\geq 0}$, and on the other hand we also find

$$
\begin{aligned}
& u w_{0}^{\prime}\left(t,-s \beta_{\mathrm{KRS}}\right)-u_{0}\left(-s \beta_{\mathrm{KRS}}\right) \\
= & \log \left(\frac{\sum_{i=1}^{m} \mathrm{e}^{\left\langle p^{(i)},-s \beta_{\mathrm{KRS}}-s_{t} \beta_{\mathrm{KRS}}\right\rangle-s_{t} \max \left(\left\langle p^{(i)},-\beta_{\mathrm{KRS}}\right\rangle\right)}}{\sum_{i=1}^{m} \mathrm{e}^{\left\langle p^{(i)},-s \beta_{\mathrm{KRS}}\right\rangle}}\right) \\
\geq & \log \left(\frac{\left(\sharp \mathrm{L} P_{\max }^{*}\right) \cdot \mathrm{e}^{s \max \left(\left\langle p^{(i)},-\beta_{\mathrm{KRS}}\right\rangle\right)}}{\sum_{i=1}^{m} \mathrm{e}^{\left\langle p^{(i)},-s \beta_{\mathrm{KRS}}\right\rangle}}\right) \\
\rightarrow & 0
\end{aligned}
$$

as $s \rightarrow \infty$. From (3.5) and (3.6), we find

$$
\lim _{s \rightarrow \infty}\left(u_{0}^{\prime}\left(t,-s \beta_{\mathrm{KRS}}\right)-u_{0}\left(-s \beta_{\mathrm{KRS}}\right)\right)=0
$$

From (3.5) and (3.7) we find $\sup _{x \in N_{\mathbb{R}}}\left(u_{0}^{\prime}(t, x)-u_{0}(x)\right)=0$, that is to say, $\psi_{t}(x)=u_{0}^{\prime}(t, x)-u_{0}(x)$.

Theorem 3.1. Let $\delta^{*}$ be an $(n-l-1)$-dimensional face of $P^{*}$. Let $\delta$ is the $l$-dimensional face of $P$ associated with $\delta^{*}$. Then, we have the following two possibilities;

(i) If for any $x \in \delta$ there is an integral point $p_{x} \in \mathrm{L} P_{\max }^{*}$ such that $\left\langle p_{x}, x\right\rangle \geq$ 0 , then $c_{\left\{p^{\left(\delta^{*}\right)}\right\}}\left(\psi_{\infty}\right) \geq 1$. In particular $\mu^{-1}\left(\delta^{*}\right)$ is not contained in the support of the MIS of $\mathcal{I}\left(\gamma \psi_{\infty}\right)$ for any $\gamma<1$.

(ii) Suppose that there is a point $x \in \delta$ such that

$$
\langle p, x\rangle<0 \text { for any } p \in \mathrm{L} P_{\max }^{*}
$$


Let $p_{\max } \in \mathrm{L} P_{\max }^{*}$ and $x^{(0)}$ be a point in $\delta$ such that

$$
\left\langle p_{\max }, x^{(0)}\right\rangle=\min _{x} \max _{p \in \mathrm{L} P_{\max }^{*}}\langle p, x\rangle
$$

where $x$ runs over $\{x \in \delta \mid x$ satisfies (3.8) $\}$. Then, we have

$$
c_{\left\{p^{\left(\delta^{*}\right)}\right\}}\left(\psi_{\infty}\right)=\frac{1}{1-\left\langle p_{\max }, x^{(0)}\right\rangle}<1 .
$$

In particular, $\mu^{-1}\left(\delta^{*}\right)$ is contained in the support of the MIS of $\mathcal{I}\left(\gamma \psi_{\infty}\right)$ for any $\gamma \in\left(c_{\left\{p^{\left(\delta^{*}\right)}\right\}}\left(\psi_{\infty}\right), 1\right)$.

Proof. First, we shall show the case (i). For any $x \in \delta$, the assumption implies

$$
u_{0}^{\prime}(t, s x) \geq s\left\langle p_{x}, x\right\rangle \geq 0
$$

for all $s \geq 0$. Since there exists a constant $C$ independent of $t$ and $x$ such that

$$
\left|u_{0}^{\prime}(t, y)-u_{0}^{\prime}(t, x)\right| \leq C, \quad \text { for any } y \in B_{1}(x),
$$

where $B_{1}(x)=\left\{y \in N_{\mathbb{R}}|| x-y \mid<1\right\}$, equation (3.10) implies that $u_{0}^{\prime}(t, \cdot)$ is bounded uniformly from below over

$$
\tilde{U}:=\left\{s_{1} x+s_{2} \eta \in N_{\mathbb{R}}\left|x \in \delta, \eta \in N_{\mathbb{R}},\right| \eta\left|=1, s_{i} \in \mathbb{R}_{\geq 0},\right| s_{2} \mid<1\right\}
$$

Let $U_{\underline{p}^{\left(\delta^{*}\right)}} \subset X$ be the interior of $\mu^{-1}(\overline{\tilde{\mu}(\tilde{U})})$, where $\overline{\tilde{\mu}(\tilde{U})}$ denotes the closure of $\tilde{\mu}(\tilde{U})$. Then, from (3.2), we find that $U_{p^{\left(\delta^{*}\right)}}$ is an open neighborhood around $\mu^{-1}\left(p^{\left(\delta^{*}\right)}\right)$. Then, for $c \geq 0$,

$$
\begin{aligned}
\int_{U_{p}\left(\delta^{*}\right)} \mathrm{e}^{-c \psi_{t}} \omega_{0}^{n} & \leq C \int_{\tilde{U}} \mathrm{e}^{-c \psi_{t}-u_{0}} d x \\
& =C \int_{\tilde{U}} \mathrm{e}^{-c u_{0}^{\prime}(t, x)+(-1+c) u_{0}(x)} d x \\
& \leq C \int_{\tilde{U}} \mathrm{e}^{(-1+c) u_{0}(x)} d x \\
& \leq C\left(\int_{s=0}^{\infty} \mathrm{e}^{(-1+c) s} d s\right)^{l+1} .
\end{aligned}
$$


In (3.11), we use the fact (cf. Lemma 4.3 in [23]) that $\mathrm{e}^{u_{0}} \operatorname{det}\left(\frac{\partial^{2} u_{0}}{\partial x_{i} \partial x_{j}}\right)$ is bounded. From (3.13), we find that $\int_{U_{\left.p^{\left(\delta^{*}\right.}\right)}} \mathrm{e}^{-c \psi_{t}} \omega_{0}^{n}$ is bounded if $0 \leq c<1$. Hence we find that $c_{\left\{p^{\left(\delta^{*}\right)}\right\}}\left(\psi_{\infty}\right) \geq 1$.

Next we shall prove the case (ii). Before proving it, remark that the existence of the points $p_{\max }$ and $x^{(0)}$ in (3.9) is assured. In fact a function $x \mapsto \max _{p \in \mathrm{L} P_{\max }^{*}}\langle p, x\rangle$ is continuous on a compact set $\{x \in \delta \mid\langle p, x\rangle \leq$ 0 for all $\left.p \in \mathrm{L} P_{\max }^{*}\right\}$ and it is

$$
\left\{\begin{array}{cl}
\text { equal to zero } & \text { if } \quad\langle p, x\rangle=0 \text { for some } p \in \mathrm{L} P_{\max }^{*} \\
\text { strictly less than zero } & \text { if } \quad\langle p, x\rangle<0 \text { for all } p \in \mathrm{L} P_{\max }^{*}
\end{array}\right.
$$

These mean that the minimal point $x^{(0)}$ of the above function is contained in $\left\{x \in \delta \mid\langle p, x\rangle<0\right.$ for all $\left.p \in \mathrm{L} P_{\max }^{*}\right\}$. Let us begin to prove (ii). The definition (3.9) implies that for all $x \in \delta$

$$
u_{0}^{\prime}(t, s x) \geq s \max _{p \in \mathrm{L} P_{\max }^{*}}\langle p, x\rangle \geq s\left\langle p_{\max }, x^{(0)}\right\rangle .
$$

Since for any $x \in \delta$ there is a vertex $p$ of $P^{*}$ such that $\langle x, p\rangle=1$, then we have

$$
u_{0}(s x) \geq s
$$

As (3.12), for $0 \leq c<1,(3.14)$ and (3.15) imply

$$
\begin{aligned}
\int_{U_{p^{\left(\delta^{*}\right)}}} \mathrm{e}^{-c \psi_{t}} \omega_{0}^{n} & \leq C \int_{\tilde{U}} \mathrm{e}^{-c u_{0}^{\prime}(t, x)+(-1+c) u_{0}(x)} d x \\
& \leq C\left(\int_{s=0}^{\infty} \mathrm{e}^{s\left\{-1+c\left(1-\left\langle p_{\max }, x^{(0)}\right\rangle\right)\right\}} d s\right)^{l+1} .
\end{aligned}
$$

From (3.16) we find that

$$
c_{\left\{p^{\left(\delta^{*}\right)}\right\}}\left(\psi_{\infty}\right) \geq \frac{1}{1-\left\langle p_{\max }, x^{(0)}\right\rangle} .
$$

Next we shall prove $c_{\left\{p^{\left(\delta^{*}\right)}\right\}}\left(\psi_{\infty}\right) \leq \frac{1}{1-\left\langle p_{\max }, x^{(0)}\right\rangle}$. For each $p^{(i)} \in \mathrm{L} P^{*}$, let

$$
A_{i}(s):=\left\langle p^{(i)}, s x^{(0)}-s_{t} \beta_{\mathrm{KRS}}\right\rangle-s_{t}\left\langle p_{\mathrm{max}},-\beta_{\mathrm{KRS}}\right\rangle
$$


Then by (3.4), for all $i=1, \ldots, m$, we have

$$
\begin{aligned}
A_{i}(s)= & s\left(\left\langle p_{\max }, x^{(0)}\right\rangle+\left\langle p^{(i)}-p_{\max }, x^{(0)}\right\rangle\right) \\
& -s_{t}\left(\left\langle p^{(i)}, \beta_{\mathrm{KRS}}\right\rangle-\left\langle p_{\max }, \beta_{\mathrm{KRS}}\right\rangle\right) \\
\leq & s\left(\left\langle p_{\max }, x^{(0)}\right\rangle+\left\langle p^{(i)}-p_{\max }, x^{(0)}\right\rangle\right) .
\end{aligned}
$$

Let us consider the following two possibilities separately.

$$
\left\{\begin{array}{l}
\text { (a) }\left\langle p^{(i)}-p_{\max }, x^{(0)}\right\rangle \leq 0 \\
\text { (b) }\left\langle p^{(i)}-p_{\max }, x^{(0)}\right\rangle>0 .
\end{array}\right.
$$

In the case (a), we have

$$
A_{i}(s) \leq s\left\langle p_{\max }, x^{(0)}\right\rangle, \quad \text { for all } s \geq 0 .
$$

Let us consider the case (b). If $p^{(i)}$ would be contained in $\mathrm{L} P_{\max }^{*},(3.9)$ induces that

$$
\left\langle p_{\max }, x^{(0)}\right\rangle=\max _{p \in \mathrm{L} P_{\max }^{*}}\left\langle p, x^{(0)}\right\rangle \geq\left\langle p^{(i)}, x^{(0)}\right\rangle
$$

This is a contradiction. Therefore, we have $p^{(i)} \notin \mathrm{L} P_{\max }^{*}$. This and (3.4) imply that $\left\langle p^{(i)}, \beta_{\mathrm{KRS}}\right\rangle-\left\langle p_{\max }, \beta_{\mathrm{KRS}}\right\rangle$ is strictly bigger than zero. Then,

$$
s\left\langle p^{(i)}-p_{\max }, x^{(0)}\right\rangle-s_{t}\left(\left\langle p^{(i)}, \beta_{\mathrm{KRS}}\right\rangle-\left\langle p_{\max }, \beta_{\mathrm{KRS}}\right\rangle\right) \leq 0
$$

for all $s \in\left[0, s_{t} T_{i}^{\prime}\right]$. Here

$$
T_{i}^{\prime}:=\frac{\left\langle\left(p^{(i)}-p_{\max }\right), \beta_{\mathrm{KRS}}\right\rangle}{\left\langle\left(p^{(i)}-p_{\max }\right), x^{(0)}\right\rangle} .
$$

Then, (3.18) implies

$$
A_{i}(s) \leq s\left\langle p_{\max }, x^{(0)}\right\rangle
$$

for all $s \in\left[0, s_{t} T_{i}^{\prime}\right]$. Let $T^{\prime}:=\min \left\{T_{i}^{\prime} \mid i=1, \ldots, m\right\}>0$. This constant depends only on $\beta_{\mathrm{KRS}}$ and independent of $s$ and $i$. Therefore, (3.19) and (3.20) imply that for all $i=1, \ldots, m$

$$
A_{i}(s) \leq s\left\langle p_{\max }, x^{(0)}\right\rangle, \quad \text { for all } s \in\left[0, s_{t} T^{\prime}\right]
$$

Let $\tilde{U}_{\varepsilon}:=\left\{x \in N_{\mathbb{R}}|| x-s x^{(0)} \mid<\varepsilon, s \geq \frac{1}{\varepsilon}\right\}$. For any open neighborhood $U^{\prime}$ of $\mu^{-1}\left(p^{\left(\delta^{*}\right)}\right)$, there is a sufficiently small constant $\varepsilon>0$ such that $\tilde{\mu}\left(\tilde{U}_{\varepsilon}\right) \subset$ 
$\mu\left(U^{\prime}\right)$. In fact, for the point $x^{(0)}$ in (3.9) we have

$$
\begin{aligned}
\frac{\partial u_{0}}{\partial x_{j}}\left(s x^{(0)}+\eta\right) & =\frac{1}{\sum_{i=1}^{m} \mathrm{e}^{\left\langle p^{(i)}, s x^{(0)+\eta}\right\rangle}}\left\{\sum_{i=1}^{m} p_{j}^{(i)} \mathrm{e}^{\left\langle p^{(i)}, s x^{(0)+\eta}\right\rangle}\right\} \\
& \rightarrow \frac{\sum_{i_{\alpha} \in A} \mathrm{e}^{\left\langle p^{\left(i_{\alpha}\right)}, \eta\right\rangle} p_{j}^{\left(i_{\alpha}\right)}}{\sum_{i_{\alpha} \in A} \mathrm{e}^{\left\langle p^{\left(i_{\alpha}\right)}, \eta\right\rangle}}
\end{aligned}
$$

as $s \rightarrow \infty$, where $A$ is the subset of $\{1, \ldots, m\}$ defined by (3.2). Since $A$ is independent of $\eta$, there is a positive constant $C$ independent of $\varepsilon$ and $\eta$ such that

$$
\begin{aligned}
& \left|\lim _{s \rightarrow \infty} \tilde{\mu}\left(s x^{(0)}+\eta\right)-p^{\left(\delta^{*}\right)}\right|^{2} \\
& =\sum_{j=1}^{n}\left|\frac{\sum_{i_{\alpha} \in A} \mathrm{e}^{\left\langle p^{\left(i_{\alpha}\right)}, \eta\right\rangle} p_{j}^{\left(i_{\alpha}\right)}}{\sum_{k_{\alpha} \in A} \mathrm{e}^{\left\langle^{\left(k_{\alpha}\right)}, \eta\right\rangle}}-\frac{\sum_{i_{\alpha} \in A} p_{j}^{\left(i_{\alpha}\right)}}{\sharp A}\right|^{2} \\
& =C \sum_{j=1}^{n} \frac{\left|\sum_{i_{\alpha} \in A}\left(\sum_{k_{\alpha} \in A}\left(\mathrm{e}^{\left\langle p^{\left(i_{\alpha}\right)}, \eta\right\rangle}-\mathrm{e}^{\left\langle p^{\left(k_{\alpha}\right)}, \eta\right\rangle}\right)\right) p_{j}^{\left(i_{\alpha}\right)}\right|^{2}}{\sharp A\left\{\sum_{k_{\alpha} \in A} \mathrm{e}^{\left.\hat{p}^{\left(k_{\alpha}\right)}, \eta\right\rangle}\right\}} \\
& \leq C \varepsilon^{2},
\end{aligned}
$$

for any sufficiently small $\varepsilon>0$ and any $\eta \in N_{\mathbb{R}}$ with $|\eta|<\varepsilon$. The inequality (3.22) follows from that for sufficiently small $\varepsilon$ there exists positive constants $c$ and $C$ such that

$$
c<\sum_{k_{\alpha} \in A} \mathrm{e}^{\left\langle p^{\left(k_{\alpha}\right)}, \eta\right\rangle}, \quad\left|\mathrm{e}^{\left\langle p^{\left(i_{\alpha}\right)}, \eta\right\rangle}-\mathrm{e}^{\left\langle p^{\left(k_{\alpha}\right)}, \eta\right\rangle}\right|^{2} \leq C|\eta|^{2} \leq C \varepsilon^{2}
$$

for any $i_{\alpha}, k_{\alpha} \in A$. Then, we find that there is a positive constant $C$ independent of $s$ and $\eta$ such that

$$
\left|\tilde{\mu}\left(s x^{(0)}+\eta\right)-\tilde{\mu}\left(s x^{(0)}\right)\right| \leq C \varepsilon,
$$

for all $s \in \mathbb{R}_{\geq 0}$ and any $\eta$ with $|\eta|<\varepsilon$. This implies that $\tilde{\mu}\left(\tilde{U}_{\varepsilon}\right) \subset \mu\left(U^{\prime}\right)$ for any sufficiently small $\varepsilon$. Remark that $\tilde{\mu}\left(\tilde{U}_{\varepsilon}\right)$ is not necessarily a neighborhood of $p^{\left(\delta^{*}\right)}$. (For instance, when $\delta^{*}$ is a 0 -dimensional face, $\tilde{\mu}\left(s x^{(0)}+\eta\right)$ goes to the point $p^{\left(\delta^{*}\right)}$ for any $\eta$, because $\sharp A=1$.) There is a positive constant $C_{\varepsilon}$ depending only on $\varepsilon$ such that, for any $x \in \tilde{U}_{\varepsilon}$ with $\left|x-s x^{(0)}\right|<\varepsilon$,

$$
u_{0}^{\prime}(t, x) \leq u_{0}^{\prime}\left(t, s x^{(0)}\right)+C_{\varepsilon}=\log \left(\sum_{i}^{m} \exp A_{i}(s)\right)+C_{\varepsilon} .
$$


On the other hand,

$$
u_{0}(s x) \leq s+\log m
$$

where $x \in \delta$ and $m$ is the number of lattice points in $P^{*}$. From (3.21), (3.24) and (3.25) we find that for $0 \leq c<1$ and a fixed sufficiently small $\varepsilon$,

$$
\begin{aligned}
& \int_{U^{\prime}} \mathrm{e}^{-c \psi_{t}} \omega_{0}^{n} \geq C \int_{\mu^{-1}\left(\tilde{\mu}\left(\tilde{U}_{\varepsilon}\right)\right)} \mathrm{e}^{-c \psi_{t}} \omega_{0}^{n} \\
& \quad \geq C \int_{\tilde{U}_{\varepsilon}} \mathrm{e}^{-c u_{0}^{\prime}(t, x)+(-1+c) u_{0}} d x \geq C \int_{\frac{1}{\varepsilon}}^{s_{t} T^{\prime}} \mathrm{e}^{-c \max _{i} A_{i}(s)+(-1+c) s} d s \\
& \geq C \int_{\frac{1}{\varepsilon}}^{s_{t} T^{\prime}} \mathrm{e}^{s\left\{c\left(1-\left\langle p_{\max }, x^{(0)}\right\rangle\right)-1\right\}} d s .
\end{aligned}
$$

If $c \geq \frac{1}{1-\left\langle p_{\max }, x^{(0)}\right\rangle}$, the RHS of (3.26) goes to $+\infty$ as $t \rightarrow \infty$, because $s_{t}$ goes to $+\infty$. The semi-continuity of the complex singularity exponent $([7])$ implies that

$$
c_{\left\{p^{\left(\delta^{*}\right)}\right\}}\left(\psi_{\infty}\right) \leq \frac{1}{1-\left\langle p_{\max }, x^{(0)}\right\rangle} .
$$

Hence we get the desired equation from (3.17) and (3.27). The proof is completed.

Remark 3.1. Theorem 3.1 is kind of a local version of Song's formula [23] of the $\alpha$-invariant on toric Fano manifolds.

Then, Lemma 3.1 is a corollary of Theorem 3.1 .

Proof of Lemma 3.1. The behavior of $s_{t}$ does not affect Theorem 3.1 and its proof. Hence, the support of the MIS of $\mathcal{I}\left(\gamma \psi_{\infty}\right)$ and of $\mathcal{I}\left(\gamma \bar{\varphi}_{\infty}\right)$ coincide.

Let $\varepsilon>0$ be a sufficiently small constant. Theorem 3.1 gives us a way to determine the support of the MIS of exponent $\gamma$ from any one-parameter subgroup of $\operatorname{Aut}(X)$ for $\gamma \in(1-\varepsilon, 1)$ as follows. Before describing this, let us introduce some terminologies. Let $\sigma_{t}$ be a one-parameter subgroup of the holomorphic vector field $v_{\zeta}$ which is associated with a vector $\zeta \in N_{\mathbb{R}}$. Let us consider the MIS coming from $\left\{\left(\sigma_{t}^{-1}\right)^{*} \omega_{0}\right\}_{t}$ as before. For distinct points $x^{(1)}$ and $x^{(2)}$ on $\partial P$, we define that $x^{(1)} \sim x^{(2)}$ if and only if $x^{(1)}$ and $x^{(2)}$ are contained in a common $(n-1)$-dimensional facet of $P$. For a point $x \in \partial P$, 
we define the star set of $x$ by

$$
\operatorname{st}(x):=\{y \in \partial P \mid y \sim x\}
$$

Let $x(-\zeta) \in \partial P$ be a point, which is the intersection between $\partial P$ and the half line $\left\{-s \zeta \in N_{\mathbb{R}} \mid s \geq 0\right\}$. Then, $s t(x(-\zeta))$ is a union of $(n-1)$-dimensional facets $\left\{\delta_{k}\right\}_{k=1, \ldots, k_{\zeta}}$ of $P$. For each $\delta_{k}$, there corresponds to a hyperplane $\left\{x \in N_{\mathbb{R}} \mid H_{k}(x)=1\right\}$ in $N_{\mathbb{R}}$, which contains $\delta_{k}$. Then, the star set $\operatorname{st}(x(-\zeta))$ divides $N_{\mathbb{R}}$ into two. This means that $N_{\mathbb{R}}$ is divided into $N_{\mathbb{R}}^{\leq}:=\left\{x \mid H_{k}(x) \leq\right.$ 1 for all $k\}$ and its complement. Then, by translating $N_{\mathbb{R}}^{\leq}$along the line $\left\{-s \zeta \in N_{\mathbb{R}} \mid s \in \mathbb{R}\right\}$ so that the origin is contained in its boundary, we define

$$
s t(\widetilde{x(-\zeta)}):=\left\{x \in N_{\mathbb{R}} \mid H_{k}(x) \leq 0, \text { for all } k\right\}
$$

Corollary 3.1. Let $X$ be a toric Fano manifold. Let $\sigma_{t}$ be a one-parameter subgroup of the holomorphic vector field $v_{\zeta}$ which is associated with a vector $\zeta \in N_{\mathbb{R}}$. Suppose $\gamma \in(1-\varepsilon, 1)$ where $\varepsilon$ is a sufficiently small positive constant. Let $\delta^{*}$ be an $(n-l-1)$-dimensional face of $P^{*}$ and $\delta$ be its associated l-dimensional face. Then, $\delta^{*}$ is contained in the image of the support of the MIS of exponent $\gamma$ from $\left\{\left(\sigma_{t}^{-1}\right)^{*} \omega_{0}\right\}_{t}$ under the moment map $\mu$ if and only if $\delta \cap \operatorname{int}(\operatorname{st}(x(-\zeta))) \neq \emptyset$, where int $($ st $(x(-\zeta)))$ is the interior of st $(x(-\zeta))$.

Proof. From the duality of $P$ and $P^{*}$, we find that for each $H_{k}$ there is a point $p^{(k)} \in \mathrm{L} P_{\max }^{*}$ corresponding to it and, that $s t(x(-\zeta))$ is equal to

$$
\left\{x \in N_{\mathbb{R}} \mid\left\langle p^{(k)}, x\right\rangle=1, \quad \text { for all } 1 \leq k \leq k_{\zeta}\right\}
$$

Hence we find that $\operatorname{int}(\widehat{s t} \widetilde{(x(-\zeta))})$ is equal to

$$
\left\{x \in N_{\mathbb{R}} \mid\left\langle p^{(k)}, x\right\rangle<0, \quad \text { for all } 1 \leq k \leq k_{\zeta}\right\}
$$

If $\delta \cap \operatorname{int}(\widetilde{s t(x(-\zeta)})) \neq \emptyset$, then Theorem 3.1 (ii) implies $c_{\left(p^{\delta^{*}}\right)}\left(\psi_{\infty}\right)<1-$ $\varepsilon(\delta)$ for some $\varepsilon(\delta)>0$ which might depend on $\delta$. By taking a sufficiently small $\varepsilon$, we get that $c_{\left(p^{\delta^{*}}\right)}\left(\psi_{\infty}\right)<1-\varepsilon$ if $\delta \cap \operatorname{int}(\widehat{s t(x(-\zeta))}) \neq \emptyset$, because the number of faces in $P$ is finite. On the other hand, if $\delta \cap \operatorname{int}(\overline{\operatorname{st}(x(-\zeta))})=\emptyset$, then Theorem 3.1 (i) implies $c_{\left(p^{\delta^{*}}\right)}\left(\psi_{\infty}\right) \geq 1 \geq 1-\varepsilon$. This completes the proof. 


\section{Examples}

In this section, we shall calculate several examples that are contained in $\mathcal{W}_{1}$. Let $v_{\text {KRS }}$ be the holomorphic vector field of Kähler-Ricci soliton, which is contained in the reductive part $\mathfrak{h}_{r}(X)$ of the Lie algebra $\mathfrak{h}(X)$ consisting of all holomorphic vector fields on $X$. Since manifolds are contained in $\mathcal{W}_{1}$, we can determine the vector $\beta_{\mathrm{KRS}}$ in $N_{\mathbb{R}}$ which induces $v_{\mathrm{KRS}}$ by calculating the sign of its Futaki invariant. Futaki invariant [11] is a Lie character of $\mathfrak{h}(X)$, defined by

$$
F(v):=\int_{X} v h_{g} \omega_{g}^{n} .
$$

It is proved that $F$ is independent of the choice of $g$. Let ( $\left.v_{\mathrm{KRS}}, \omega_{\mathrm{KRS}}\right)$ be a Kähler-Ricci soliton. It is not difficult to see

$$
F\left(v_{\mathrm{KRS}}\right)>0 .
$$

Then, in order to determine $\beta_{\text {KRS }}$ under the assumption that $X$ is contained in $\mathcal{W}_{1}$, it is sufficient to calculate the sign of Futaki invariant of the holomorphic vector field coming from a vector in $N_{\mathbb{R}}$, which is invariant under $\mathcal{W}(X)$. To calculate it, we shall use the following result;

Theorem 4.1 (Mabuchi [14]). Let $\mathbb{F}:=\left(F\left(t_{1} \frac{\partial}{\partial t_{1}}\right), \ldots, F\left(t_{n} \frac{\partial}{\partial t_{n}}\right)\right) \in \mathbb{R}^{n}$. Remark that $t_{i} \frac{\partial}{\partial t_{i}}$ is a $T_{\mathbb{C}}$-invariant holomorphic vector field on $T_{\mathbb{C}}$, which can be extended on $X$. Let $b\left(P^{*}\right) \in M_{\mathbb{R}}$ be the barycenter of $P^{*}$, i.e.,

$$
\frac{1}{\int_{P^{*}} d y}\left(\int_{P^{*}} y_{1} d y, \ldots, \int_{P^{*}} y_{n} d y\right)
$$

where $d y=d y_{1} \wedge \cdots \wedge d y_{n}$. Then $\mathbb{F}$ is equal to $-b\left(P^{*}\right)$.

The minus sign of $b\left(P^{*}\right)$ above comes from that our choice of affine logarithmic coordinates has the opposite sign to the one in [14]. Combining (4.1) and Theorem 4.1 we find

$$
\left\langle b\left(P^{*}\right), \beta_{\mathrm{KRS}}\right\rangle<0 .
$$

\subsection{Toric Fano 2-folds}

There are five types of toric Fano 2-folds; $\mathbb{C P}^{2}, \mathbb{C P}^{1} \times \mathbb{C P}^{1}$ and the blowup of $\mathbb{C P}^{2}$ at $k$ points, where $k=1,2,3$. Kähler-Einstein manifolds among 
them are $\mathbb{C P}^{2}, \mathbb{C P}^{1} \times \mathbb{C P}^{1}$ and the blow up of $\mathbb{C P}^{2}$ at 3 points. Meanwhile the blow up of $\mathbb{C P}^{2}$ at $k$ points $(k=1,2)$ does not admit Kähler-Einstein metrics and it is contained in $\mathcal{W}_{1}$. So we can apply our results to them. First, let us consider the blow up of $\mathbb{C P}^{2}$ at one point.

Example 4.1. The support of the KRF-MIS on $\mathbb{C P}^{2} \sharp \overline{\mathbb{C P}^{2}}$ of exponent $\gamma$ is the exceptional divisor for all $\gamma \in\left(\frac{1}{2}, 1\right)$.

Proof. The polytope in $N_{\mathbb{R}}$ whose vertices are

$$
(-1,-1),(-1,0),(0,-1),(1,1)
$$

corresponds to the Fano polytope $P$ of $\mathbb{C P}^{2} \sharp \overline{\mathbb{C P}^{2}}$. Then, $N_{\mathbb{R}}^{\mathcal{W}(X)}$ is the onedimensional subspace of $N_{\mathbb{R}}$ generated by a vector $(-1,-1)$ and $\beta_{\mathrm{KRS}}$ is proportional to $(-1,-1)$. Since the vertices of the polytope $P^{*}$ are

$$
(-1,0),(0,-1),(2,-1),(-1,2),
$$

it is easy to see that $\left\langle b\left(P^{*}\right),(1,1)\right\rangle>0$. Then, (4.2) implies that $\beta_{\mathrm{KRS}}=$ $\beta(-1,-1)$ where $\beta>0$. Also we find that

$$
\left.s t\left(\widetilde{x\left(-\beta_{\mathrm{KRS}}\right.}\right)\right)=\left\{x=\left(x_{1}, x_{2}\right) \mid x_{1}-2 x_{2} \geq 0,2 x_{1}-x_{2} \leq 0\right\} .
$$

The vertex of $P$ contained in $\left.\operatorname{int}\left(\operatorname{st}\left(\widetilde{x\left(-\beta_{\mathrm{KRS}}\right.}\right)\right)\right)$ is $(-1,-1)$ which represents the exceptional divisor. Then, Corollary 3.1 implies that the support of the KRF-MIS of exponent $\gamma$ is the exceptional divisor where $\gamma$ is strictly smaller than 1 and sufficiently close to 1 . $L P_{\max }^{*}$ is

$$
\{(2,-1),(-1,2)\} \text {. }
$$

For the facet $\delta^{*}$ of $P^{*}$ associated with the vertex $(-1,-1)$ of $P$,

$$
\left\langle p_{\max }, x^{(0)}\right\rangle=\langle(2,-1),(-1,-1)\rangle=\langle(-1,2),(-1,-1)\rangle=-1 .
$$

Hence,

$$
c_{\left\{p^{\left(\delta^{*}\right)}\right\}}\left(\psi_{\infty}\right)=c_{\left\{\left(-\frac{1}{2},-\frac{1}{2}\right)\right\}}\left(\psi_{\infty}\right)=\frac{1}{2} .
$$

Therefore, the proof is completed.

Next, let us consider the blow up of $\mathbb{C P}^{2}$ at $p_{1}$ and $p_{2}$. Let $E_{1}$ and $E_{2}$ be the exceptional divisors of the blow up. In $X$, there is another $(-1)$-curve denoted by $E_{0}$, which intersects with $E_{1}$ and $E_{2}$ Remark that $E_{0}$ is the proper transform of $\overline{p_{1} p_{2}}$ of the line passing through $p_{1}$ and $p_{2}$. Then, 
Example 4.2. The support of the KRF-MIS on $\mathbb{C P}^{2} \sharp 2 \overline{\mathbb{C P}^{2}}$ of exponent $\gamma$ is

$$
\left\{\begin{array}{cl}
\cup_{i=0}^{2} E_{i}, & \text { for } \gamma \in\left(\frac{1}{2}, 1\right), \\
E_{0}, & \text { for } \gamma \in\left(\frac{1}{3}, \frac{1}{2}\right) .
\end{array}\right.
$$

Proof. The polytope in $N_{\mathbb{R}}$ whose vertices are

$$
(-1,0),(0,-1),(1,0),(1,1),(0,1)
$$

corresponds to the Fano polytope $P$ of $\mathbb{C P}^{2} \sharp 2 \overline{\mathbb{C P}^{2}}$. Then, $N_{\mathbb{R}}^{\mathcal{W}(X)}$ is the onedimensional subspace of $N_{\mathbb{R}}$ generated by a vector $(1,1)$ and $\beta_{\mathrm{KRS}}$ is proportional to $(1,1)$. Since the vertices of the polytope $P^{*}$ are

$$
(-1,-1),(-1,1),(0,1),(1,0),(1,-1),
$$

we find that $\left\langle b\left(P^{*}\right),(1,1)\right\rangle<0$. Then, (4.2) implies that $\beta_{\mathrm{KRS}}=\beta(1,1)$ where $\beta>0$. Also we find that

$$
\left.\operatorname{st}\left(\widetilde{x\left(-\beta_{\mathrm{KRS}}\right.}\right)\right)=\left\{x=\left(x_{1}, x_{2}\right) \mid x_{1}+x_{2} \geq 0\right\} .
$$

The vertices of $P$ contained in $\left.\operatorname{int}\left(s t\left(\widetilde{x\left(-\beta_{\mathrm{KRS}}\right.}\right)\right)\right)$ are $(1,0),(0,1)$, which represent the exceptional divisors $E_{1}$ and $E_{2}$, and $(1,1)$, which represents the proper transform $E_{0}$. Then, Corollary 3.1 implies that the support of the KRF-MIS of exponent $\gamma$ is the sum of $E_{0}, E_{1}$ and $E_{2}$ where $\gamma$ is strictly smaller than 1 and sufficiently close to $1 . L P_{\max }^{*}$ is

$$
\{(-1,-1)\} \text {. }
$$

For the facets $\eta_{i}^{*},(i=1,2)$ of $P^{*}$ associated with the vertices $(1,0)$ and $(0,1)$ of $P$, respectively,

$$
\left\langle p_{\max }, x^{(0)}\right\rangle=\langle(-1,-1),(1,0)\rangle=\langle(-1,-1),(0,1)\rangle=-1 .
$$

Hence,

$$
c_{\left\{p^{\left.\left(\eta_{1}^{*}\right)\right\}}\right.}\left(\psi_{\infty}\right)=c_{\left\{\left(1,-\frac{1}{2}\right)\right\}}\left(\psi_{\infty}\right)=\frac{1}{2} .
$$

Also $c_{\left\{p^{\left(\eta_{2}^{*}\right)}\right\}}\left(\psi_{\infty}\right)=\frac{1}{2}$. For the facet $\delta^{*}$ associated with the vertex $(1,1)$ of $P$,

$$
\left\langle p_{\max }, x^{(0)}\right\rangle=\langle(-1,-1),(1,1)\rangle=-2 .
$$

Hence,

$$
c_{\left\{p^{\left(\delta^{*}\right)}\right\}}\left(\psi_{\infty}\right)=c_{\left\{\left(\frac{1}{2}, \frac{1}{2}\right)\right\}}\left(\psi_{\infty}\right)=\frac{1}{3}
$$

Therefore, the proof is completed. 


\subsection{Toric Fano 3-folds}

Toric Fano 3-folds are classified completely (Remark 2.5.10 in [1]). According to the classification, there are eighteen types of toric Fano 3-folds. Five of them are Kähler-Einstein manifolds, and eight of them are contained in $\mathcal{W}_{1}$ and do not admit Kähler-Einstein metrics. (As for the classification of Kähler-Einstein toric 3-folds, see [14].) In this section, we give the list of the support of the KRF-MIS for the eight examples, computed by applying our method. The data of the list consists of (a) the vertices of the Fano polytope $P$ denoted by $\operatorname{Ver} P$ and the vertices of the moment polytope $P^{*}$ denoted by $\operatorname{Ver} P^{*}$, (b) the vector $\beta_{\mathrm{KRS}}$ and (c) the vertices of contained in $\operatorname{int}\left(\operatorname{st}\left(\widetilde{\left.x\left(-\beta_{\mathrm{KRS}}\right)\right)}\right)\right.$ denoted by $\widetilde{\operatorname{Ver} P}=\left\{q^{(i)}\right\}_{i}$ and the complex singularity exponent corresponding to $q^{(i)}$, which is denoted by $c_{i}$.

Example 4.3. Let $X_{k}$ be the blow up of $\mathbb{C P}^{n}$ along $\mathbb{C P}^{k}$, where $0 \leq k \leq$ $n-2$. The support of the KRF-MIS of complex singular exponent $\gamma$ is the exceptional divisor for $\gamma \in\left(\frac{1}{k+2}, 1\right)$.

(a)

$$
\operatorname{Ver} P=\underbrace{\left(\begin{array}{cccccc}
a_{1} & -1 & 0 & \cdots & 0 & 1 \\
a_{2} & 0 & -1 & & 0 & 1 \\
\vdots & \vdots & 0 & \ddots & 0 & \vdots \\
a_{n} & 0 & 0 & \cdots & -1 & 1
\end{array}\right)}_{n+2}
$$

where $a_{i}=-1$ for $1 \leq i \leq n-k$, otherwise $a_{i}=0$. Ver $P^{*}$ consists of

$$
\left\{p^{(i)}=\left(b_{1}^{(i)}, \ldots, b_{n}^{(i)}\right) \mid b_{i}^{(i)}=n, b_{j}^{(i)}=-1 \text { for } j \neq i\right\}_{1 \leq i \leq n-k}
$$

and

$$
\left\{p^{(i)} \in \operatorname{Ver} P^{*} \mid\left\langle p^{(i)}, q^{(1)}\right\rangle=1\right\}_{n-k+1 \leq i \leq \sharp \operatorname{Ver} P^{*}},
$$

where $q^{(1)}=\left(a_{1}, \ldots, a_{n}\right)$.

(b) $\beta_{\mathrm{KRS}}=\left(a_{1}, \ldots, a_{n}\right)$.

(c) $\widetilde{\operatorname{Ver} P}=\left\{q^{(1)}\right\}$ with

$$
c_{1}=\frac{1}{1-\left\langle p^{(i)}, q^{(1)}\right\rangle}=\frac{1}{k+2} \quad(\text { for any } 1 \leq i \leq n-k) .
$$

The vertex $q^{(1)}$ represents the exceptional divisor. 
Remark that $\mathcal{B}_{2}=\mathbb{P}_{\mathbb{C P}^{2}}(\mathcal{O} \oplus \mathcal{O}(1))$ and $\mathcal{B}_{3}=\mathbb{P}_{\mathbb{C P}^{1}}(\mathcal{O} \oplus \mathcal{O} \oplus \mathcal{O}(1))$ in [1] are contained in Example 4.3 as the case for $(n=3, k=0)$ and $(n=3, k=1)$ respectively.

Example 4.4. Let $\mathcal{B}_{1}$ be $\mathbb{P}_{\mathbb{C P}^{2}}(\mathcal{O} \oplus \mathcal{O}(2))$. The support of the KRF-MIS on $\mathcal{B}_{1}$ of exponent $\gamma$ is $S_{\infty}$ for $\gamma \in\left(\frac{1}{2}, 1\right)$. Here $S_{\infty}$ is the divisor defined by a section $(0, \sigma)$ of $\mathcal{O} \oplus \mathcal{O}(2)$ over $\mathbb{C P}^{2}$. More precisely, $S_{\infty}$ is the closure of

$$
\left\{[0 ; \sigma(p)] \in \mathcal{B}_{1} \mid \sigma(p) \neq 0\right\} .
$$

Remark that it is not an exceptional divisor.

(a)

$\operatorname{Ver} P=\left(\begin{array}{ccccc}1 & -1 & 1 & 1 & 0 \\ 0 & 0 & 1 & 0 & -1 \\ 0 & 0 & 0 & 1 & -1\end{array}\right), \quad \operatorname{Ver} P^{*}=\left(\begin{array}{cccccc}1 & 1 & 1 & -1 & -1 & -1 \\ 0 & -1 & 0 & 2 & -3 & 2 \\ 0 & 0 & -1 & 2 & 2 & -3\end{array}\right)$.

(b) $\beta_{\mathrm{KRS}}=(1,0,0)$.

(c) $\widetilde{\operatorname{Ver} P}=\left\{q^{(1)}=(1,0,0)\right\}$ with $c_{1}=\frac{1}{2}$, which represents $S_{\infty}$.

Example 4.5. Let $\mathcal{C}_{1}$ be $\mathbb{P}_{\mathbb{C P}^{1} \times \mathbb{C P}^{1}}(\mathcal{O} \oplus \mathcal{O}(1,1))$. The support of the KRFMIS on $\mathcal{C}_{1}$ of exponent $\gamma$ is $S_{\infty}$ for $\gamma \in\left(\frac{1}{2}, 1\right)$. Here $S_{\infty}$ is the divisor defined by a section $\left(0, \sigma_{1} \otimes \sigma_{2}\right)$ of $\mathcal{O} \oplus \mathcal{O}(1,1)$ over $\mathbb{C P}^{1} \times \mathbb{C P}^{1}$ and $\sigma_{i}$ is the pullback of the section of $\mathcal{O}_{\mathbb{C P}^{1}}(1)$ with respect to the $i$ th projection $\mathbb{C P}^{1} \times$ $\mathbb{C P}^{1} \rightarrow \mathbb{C P}^{1}$. Remark that $S_{\infty}$ is not an exceptional divisor.

(a)

$$
\begin{aligned}
\operatorname{Ver} P & =\left(\begin{array}{cccccc}
0 & 0 & 1 & 0 & -1 & 0 \\
0 & 0 & 0 & 1 & 0 & -1 \\
1 & -1 & 1 & 1 & 0 & 0
\end{array}\right), \\
\operatorname{Ver} P^{*} & =\left(\begin{array}{cccccccc}
0 & 0 & -1 & -1 & 2 & 2 & -1 & -1 \\
0 & -1 & 0 & -1 & -1 & 2 & 2 & -1 \\
1 & 1 & 1 & 1 & -1 & -1 & -1 & -1
\end{array}\right) .
\end{aligned}
$$

(b) $\beta_{\mathrm{KRS}}=(0,0,1)$.

(c) $\widetilde{\operatorname{Ver} P}=\left\{q^{(1)}=(0,0,1)\right\}$ with $c_{1}=\frac{1}{2}$, which represents $S_{\infty}$.

Example 4.6. Let $\mathcal{C}_{4}$ be $\left(\mathbb{C P}^{2} \sharp \overline{\mathbb{C P}^{2}}\right) \times \mathbb{C P}^{1}$, which is the blow up of $\mathbb{C P}^{2} \times$ $\mathbb{C P}^{1}$ along $\{$ point $\} \times \mathbb{C P}^{1}$. The support of the KRF-MIS on $\mathcal{C}_{4}$ of exponent $\gamma$ is the exceptional divisor of the blow up for $\gamma \in\left(\frac{1}{2}, 1\right)$. 
(a)

$$
\begin{aligned}
\operatorname{Ver} P & =\left(\begin{array}{cccccc}
0 & 0 & -1 & -1 & 1 & 0 \\
0 & 0 & -1 & 0 & 1 & -1 \\
1 & -1 & 0 & 0 & 0 & 0
\end{array}\right), \\
\operatorname{Ver} P^{*} & =\left(\begin{array}{ccccccccc}
0 & -1 & 2 & -1 & 0 & -1 & 2 & -1 \\
-1 & 2 & -1 & 0 & -1 & 2 & -1 & 0 \\
1 & 1 & 1 & 1 & -1 & -1 & -1 & -1
\end{array}\right)
\end{aligned}
$$

(b) $\beta_{\mathrm{KRS}}=(-1,-1,0)$.

(c) $\widetilde{\operatorname{Ver} P}=\left\{q^{(1)}=(-1,-1,0)\right\}$ with $c_{1}=\frac{1}{2}$, which represents the exceptional divisor.

Next, we consider a $\left(\mathbb{C P}^{2} \sharp 2 \overline{\mathbb{C P}^{2}}\right)$-bundle $\mathcal{E}_{1}$ over $\mathbb{C P}^{1}$. This manifold is derived as follows. Let $\tilde{E}_{0}$ be its exceptional divisor of the blow up $\pi: \mathcal{B}_{3} \rightarrow$ $\mathbb{C P}^{3}$ along a curve

$$
F_{0}:=\left\{\left[z_{0} ; z_{1}: 0: 0\right] \in \mathbb{C P}^{3} \mid z_{i} \in \mathbb{C}\right\} \simeq \mathbb{C P}^{1}
$$

Let $\tilde{F}_{1}$ and $\tilde{F}_{2}$ are the two $\left(T_{\mathbb{C}}\right.$-fixed $)$ curves, which are reduced to $F_{0}$ under $\pi$. Then $\mathcal{E}_{1}$ is constructed from the blow up of $\mathcal{B}_{3}$ along $\tilde{F}_{1}$ and $\tilde{F}_{2}$. Let $\tilde{\tilde{E}}_{0}$

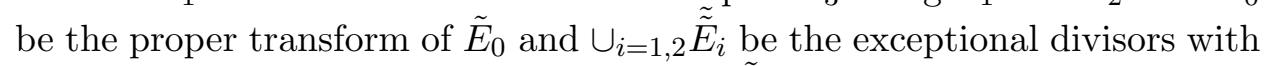
respect to the blow up of $\mathcal{B}_{3}$. Remark that $\tilde{\tilde{E}}_{0}$ is not exceptional in $\mathcal{E}_{1}$.

Example 4.7. Let $\mathcal{E}_{1}$ be a $\left(\mathbb{C P}^{2} \sharp 2 \overline{\mathbb{C P}^{2}}\right)$-bundle over $\mathbb{C P}^{1}$ defined as above. The support of the KRF-MIS on $\mathcal{E}_{1}$ of exponent $\gamma$ is

$$
\left\{\begin{array}{cl}
\tilde{\tilde{E}}_{0} \cup\left(\cup_{i=1,2} \tilde{\tilde{E}}_{i}\right), & \text { for } \gamma \in\left(\frac{1}{2}, 1\right), \\
\tilde{\tilde{E}}_{0}, & \text { for } \gamma \in\left(\frac{1}{3}, \frac{1}{2}\right) .
\end{array}\right.
$$

(a)

$$
\begin{aligned}
\operatorname{Ver} P & =\left(\begin{array}{ccccccc}
1 & 0 & -1 & 0 & 1 & 1 & 0 \\
1 & 1 & 0 & -1 & 0 & 1 & 0 \\
0 & 0 & 0 & 0 & 0 & 1 & -1
\end{array}\right) \\
\operatorname{Ver} P^{*} & =\left(\begin{array}{cccccccccc}
0 & -1 & -1 & 1 & 1 & 0 & -1 & -1 & 1 & 1 \\
1 & 1 & -1 & -1 & 0 & 1 & 1 & -1 & -1 & 0 \\
0 & 1 & 3 & 1 & 0 & -1 & -1 & -1 & -1 & -1
\end{array}\right)
\end{aligned}
$$

(b) $\beta_{\mathrm{KRS}}=(1,1,0)$. 
(c) $\widetilde{\operatorname{Ver} P}=\left\{q^{(1)}, q^{(2)}, q^{(3)}\right\}=\{(1,1,0),(0,1,0),(1,0,0)\}$, $\tilde{\tilde{E}}_{\tilde{E}}$ where $\quad$ the vertices $\left\{q^{(1)}\right\}$ and $\left\{q^{(2)}, q^{(3)}\right\}$ represent $\tilde{\tilde{E}}_{0}$ and $\left\{\tilde{\tilde{E}}_{1}, \tilde{\tilde{E}}_{2}\right\}$, respectively. Their complex singularity exponents are given by $c_{1}=\frac{1}{3}$ and $c_{2}=c_{3}=\frac{1}{2}$.

Example 4.8. Let $\mathcal{E}_{3}$ be $\left(\mathbb{C P}^{2} \sharp 2 \overline{\mathbb{C P}^{2}}\right) \times \mathbb{C P}^{1}$, which is the blow-up of $\mathbb{C P}^{1} \times$ $\mathbb{C P} P^{1} \times \mathbb{C P}^{1}$ along $\left\{p_{1}\right\} \times\left\{p_{2}\right\} \times \mathbb{C P}^{1}$. The support of the KRF-MIS on $\mathcal{E}_{3}$ of exponent $\gamma$ is

$$
\left\{\begin{array}{cl}
\cup_{i=0}^{2} E_{i}, & \text { for } \gamma \in\left(\frac{1}{2}, 1\right), \\
E_{0}, & \text { for } \gamma \in\left(\frac{1}{3}, \frac{1}{2}\right) .
\end{array}\right.
$$

Here $E_{0}$ denotes the exceptional divisor of the blow-up and $E_{1}$ (resp. $E_{2}$ ) denotes the proper transform of $\mathbb{C P}^{1} \times\left\{p_{2}\right\} \times \mathbb{C P}^{1}\left(\right.$ resp. $\left.\left\{p_{1}\right\} \times \mathbb{C P}^{1} \times \mathbb{C P}^{1}\right)$

(a)

$$
\begin{aligned}
\operatorname{Ver} P & =\left(\begin{array}{ccccccc}
1 & 0 & -1 & 0 & 1 & 0 & 0 \\
1 & 1 & 0 & -1 & 0 & 0 & 0 \\
0 & 0 & 0 & 0 & 0 & 1 & -1
\end{array}\right) \\
\operatorname{Ver} P^{*} & =\left(\begin{array}{cccccccccc}
0 & -1 & -1 & 1 & 1 & 0 & -1 & -1 & 1 & 1 \\
1 & 1 & -1 & -1 & 0 & 1 & 1 & -1 & -1 & 0 \\
1 & 1 & 1 & 1 & 1 & -1 & -1 & -1 & -1 & -1
\end{array}\right)
\end{aligned}
$$

(b) $\beta_{\mathrm{KRS}}=(1,1,0)$.

(c) $\widetilde{\operatorname{Ver} P}=\left\{q^{(1)}, q^{(2)}, q^{(3)}\right\}=\{(1,1,0),(0,1,0),(1,0,0)\}$. The vertices $\left\{q^{(1)}\right\}$ and $\left\{q^{(2)}, q^{(3)}\right\}$ represent $E_{0}$ and $\left\{E_{1}, E_{2}\right\}$ respectively. Their complex singularity exponents are given by $c_{1}=\frac{1}{3}$ and $c_{2}=c_{3}=\frac{1}{2}$.

Finally let us consider a $\left(\mathbb{C P}^{2} \sharp 3 \overline{\mathbb{C P}^{2}}\right)$-bundle $\mathcal{F}_{2}$ over $\mathbb{C P}^{1}$. Let $\tilde{E}_{0}, \tilde{\tilde{E}}_{i}$ $(i=0,1,2), F_{0}$, and $\tilde{F}_{i}(i=1,2)$ be as in Example 4.7. Let $\tilde{\pi}: \mathcal{E}_{1} \rightarrow \mathcal{B}_{3}$ be the blow up of $\mathcal{B}_{3}$ along $\tilde{F}_{1}$ and $\tilde{F}_{2}$. Let $F_{3}$ be the $\mathbb{C P}^{1}$ in $\mathbb{C P}^{3}$ defined by

$$
F_{3}:=\left\{\left[0: 0: z_{3} ; z_{4}\right] \mid z_{i} \in \mathbb{C}\right\} .
$$

The manifold $\mathcal{F}_{2}$ is constructed from the blow-up of $\mathcal{E}_{1}$ along the curve $\tilde{\pi}^{-1}\left(\pi^{-1}\left(F_{3}\right)\right)$. Let $\tilde{\tilde{E}}_{0}$ be the proper transform of $\tilde{\tilde{E}}_{0}$ with respect to the blow up of $\mathcal{E}_{1}$ along the curve $\tilde{\pi}^{-1}\left(\pi^{-1}\left(F_{3}\right)\right)$. Remark that $\tilde{\tilde{E}}_{0}$ is not an exceptional divisor. 
Example 4.9. Let $\mathcal{F}_{2}$ be a $\left(\mathbb{C P}^{2} \sharp 3 \overline{\mathbb{C P}^{2}}\right)$-bundle over $\underset{\widetilde{\widetilde{C}}}{\mathbb{C}} \mathbb{P}^{1}$ defined as above. The support of the KRF-MIS on $\mathcal{F}_{2}$ of exponent $\gamma$ is $\tilde{\tilde{E}}_{0}$ for $\gamma \in\left(\frac{1}{2}, 1\right)$.

(a)

$$
\begin{aligned}
& \operatorname{Ver} P=\left(\begin{array}{cccccccc}
1 & 0 & -1 & -1 & 0 & 1 & 1 & 0 \\
0 & 1 & 1 & 0 & -1 & -1 & 0 & 0 \\
0 & 0 & 0 & 0 & 0 & 0 & 1 & -1
\end{array}\right) \\
& \operatorname{Ver} P^{*}=\left(\begin{array}{cccccccccccc}
1 & 0 & -1 & -1 & 0 & 1 & 1 & 0 & -1 & -1 & 0 & 1 \\
1 & 1 & 0 & -1 & -1 & 0 & 1 & 1 & 0 & -1 & -1 & 0 \\
0 & 1 & 2 & 2 & 1 & 0 & -1 & -1 & -1 & -1 & -1 & -1
\end{array}\right) \text {. }
\end{aligned}
$$

(b) $\beta_{\mathrm{KRS}}=(1,0,0)$.

(c) $\widetilde{\operatorname{Ver} P}=\left\{q^{(1)}=(1,0,0)\right\}$ with $c_{1}=\frac{1}{2}$, which represents $\widetilde{\tilde{E}}_{0}$.

\section{Acknowledgments}

This work was supported by World Premier International Research Center Initiative (WPI Initiative), MEXT Japan while the author was a project researcher of the Institute for the Physics and Mathematics of the Universe (IPMU). The author thanks Professor Akito Futaki for his valuable comments for the improvement of this paper. He also thanks the referees for carefully examining this article and providing helpful comments.

\section{References}

[1] V.V. Batyrev, On the classification of toric Fano 4-folds, J. Math. Sci. 94(1) (1999), 1021-1050.

[2] V.V. Batyrev and E.N. Selivanova, Einstein-Kähler metrics on symmetric toric Fano manifolds, J. Reine Angew. Math. 512 (1999), 225-236.

[3] H.D. Cao, Deformation of Kähler metrics to Kähler-Einstein metrics on compact Kähler manifolds, Invent. Math. 81 (1985), 359-372.

[4] I. Cheltsov and C. Shramov, Log canonical thresholds of smooth Fano threefolds, Usp. Mat. Nauk 63(5)(383) (2008), 73-180.

[5] X.X. Chen and B. Wang, Remarks on Kähler-Ricci flow, J. Geom. Anal. 20(2) (2010), 335-353. 
[6] X.X. Chen and G. Tian, Ricci flow on Kähler-Einstein manifolds, Duke Math. J. 131(1) (2006), 17-73.

[7] J.P. Demailly and J. Kollár, Semi-continuity of complex singularity exponents and Kähler-Einstein Metrics on Fano Orbifolds, Ann. Sci. École Norm. Sup. (4) 34(4) (2001), 525-556.

[8] S.K. Donaldson, Scalar curvature and stability of toric varieties, J. Diff. Geom. 62(2) (2002), 289-349.

[9] S.K. Donaldson, Kähler geometry on toric manifolds, and some other manifolds with large symmetry, in Handbook of Geometric Analysis. No. 1, 29-75, Adv. Lect. Math. (ALM), 7 (2008).

[10] W. Fulton, Introduction to toric varieties, Ann. Math. Stud. 131 (1993).

[11] A. Futaki, An obstruction to the existence of Einstein-Kähler metrics, Invent. Math. 73(3) (1983), 437-443.

[12] A. Futaki and Y. Sano, Multiplier ideal sheaves and integral invariants on toric Fano manifolds, Math. Ann. 350 (2011), 245-267.

[13] G. Heier, Convergence of the Kähler-Ricci flow and multiplier ideal sheaves on Del Pezzo surfaces, Michigan Math. J. 58(2) (2009), 423-440.

[14] T. Mabuchi, Einstein-Kähler forms, Futaki invariants and convex geometry on toric Fano varieties, Osaka J. Math. 24(4) (1987), $705-737$.

[15] Y. Matsushima, Sur la structure du group d'homémomorphismes analytiques d'une certaine variété kählériennes, Nagoya Math. J. 11 (1957), $145-150$.

[16] A.M. Nadel, Multiplier ideal sheaves and Kähler-Einstein metrics of positive scalar curvature, Ann. Math. (2) 132(3) (1990), $549-596$.

[17] B. Nill and A. Paffenholz, Examples of Kähler-Einstein toric Fano manifolds associated to non-symmetric reflexive polytopes, Beitr. Algebra Geom. 52 (2011), 297-304.

[18] T. Oda, Convex bodies and algebraic geometry, Ergebnisse der Mathematik und ihrer Grenzgebiete (3), 15, Springer-Verlag, Berlin, 1988. 
[19] H. Ono, Y. Sano and N. Yotsutani, An example of asymptotically Chow unstable manifolds with constant scalar curvature, Ann. Inst. Fourier to appear (arXiv:0906.3836 (2009)).

[20] D.H. Phong, N. Sesum and J. Sturm, Multiplier ideal sheaves and the Kähler-Ricci flow, Comm. Anal. Geom. 15(3) (2007), 613-632.

[21] Y.A. Rubinstein, On the construction of Nadel multiplier ideal sheaves and the limiting behavior of the Ricci flow, Trans. Amer. Math. Soc. 361(11) (2009), 5839-5850.

[22] Y. Sano, On the holomorphic invariants for generalized Kähler-Einstein metrics, Kodai Math. J. 31 (2008), 431-440.

[23] J. Song, The $\alpha$-invariant on toric Fano manifolds, Amer. J. Math. 127(6) (2005), 1247-1259.

[24] G. Tian, On Kähler-Einstein metrics on certain Kähler manifolds with $C_{1}(M)>0$, Invent. Math. 89(2) (1987), 225-246.

[25] G. Tian and S.T. Yau, Kähler-Einstein metrics on complex surfaces with $C_{1}(M)$ positive, Comm. Math. Phys. 112 (1987), 175-203.

[26] G. Tian, Kähler-Einstein metrics with positive scalar curvature, Invent. Math. 130(1) (1997), 1-37.

[27] G. Tian and X. Zhu, A new holomorphic invariant and uniqueness of Kähler-Ricci solitons, Comment. Math. Helv. 77(2) (2002), 297-325.

[28] G. Tian and X. Zhu, Convergence of Kähler-Ricci flow, J. Amer. Math. Soc. 20(3) (2007), 675-699.

[29] X.J. Wang and X. Zhu, Kähler-Ricci solitons on toric manifolds with positive first Chern class, Adv. Math. 188(1) (2004), 87-103.

[30] S.T. Yau, On the Ricci curvature of a compact Kähler manifold and the complex Monge-Ampère equation. I, Comm. Pure Appl. Math. 31(3) (1978), 339-411.

[31] X. Zhu, Kähler-Ricci flow on a toric manifold with positive first Chern class, arXiv:math.DG/0703486 (2007). 
Graduate School of Science and Technology Kumamoto University

2-39-1, Kurokami, Kumamoto, 860-8555

JAPAN

E-mail address: sano@sci.kumamoto-u.ac.jp

Received February 1, 2012 